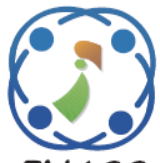

\title{
Blockchain-Based Data Sharing for Decentralized Tourism Destinations Recommendation System
}

\author{
Yunifa Miftachul Arif ${ }^{1,2} \quad$ Hani Nurhayati $^{2} \quad$ Fachrul Kurniawan $^{2}$ \\ Supeno Mardi Susiki Nugroho ${ }^{1} \quad$ Mochamad Hariadi $^{1 *}$ \\ ${ }^{l}$ Department of Electrical Engineering, Institut Teknologi Sepuluh Nopember, Surabaya, Indonesia \\ ${ }^{2}$ Department of Informatics Engineering, Universitas Islam Negeri Maulana Malik Ibrahim, Malang, Indonesia \\ * Corresponding author's Email: mochar@ee.its.ac.id
}

\begin{abstract}
One thing that tourists need to plan their tourism activities is a recommendation system. The tourism destinations recommendation system in this study has three primary nodes, namely user, server, and sensor. Each node requires the ability to share data to produce recommendations that the user expects through their mobile devices. In this paper, we propose the data-sharing system scheme uses a blockchain-based decentralized network that each node can be connected directly to each other, to support the exchange of data between them. The block architecture used in the blockchain network has three main parts, namely block information, hashes, and data. Each type of node has a different structure and direction of data communication. Where the user node sends destination assessment data to the server node, then the server node sends data from the machine learning process to the user node. The sensor sends dynamic data about popularity, traffic, and weather to the user node as consideration for finalizing the generating recommendations process. In the process of sending data, each node in the blockchain network goes through several functions, including hashing, block validation, chaining block, and broadcast. We conduct web-based experiments and analysis of the data-sharing system to illustrate the system works. The experimental results show that the system handles data circulation with an average time of mine is $84.5 \mathrm{~ms}$ in sending multi-criteria assessment data from the user and $119.1 \mathrm{~ms}$ in sending data of machine learning result from the server.
\end{abstract}

Keywords: Recommendation system, Tourism destinations, Data-sharing, Decentralized, Blockchain.

\section{Introduction}

Three stages must be passed by tourists when taking a tour called before the trip, during the trip, and after the trip [1]. The initial phase of a tour is before the trip, where people try to plan and select tourism destinations that will be visited [2]. The results of selecting destinations that are following the tourists' wishes and characteristics are essential for them [3]. Tourists can hire a guide to help to provide recommendations for destinations selection, but this can increase their spending budget [4]. To reduce the expenses, tourists must determine their own desired tourism destination. It begins with the information search for each potential tourist destination. The process can be done by utilizing the sophistication of multimedia technology, for example, through mobile equipment [5].

Mobile equipment, such as smartphones, in addition to functioning as a communication tool, is also a tool that makes exchanging information easier for tourists [6]. In the destination selection process, tourists can use the internet browser or social media applications to find the information details of the desired destination [7, 8]. However, what tourists need is not only information but more critical is assistance or recommendations, for example, in determining the selection of tourism destinations [9]. If tourists make a mistake in the tourism destinations choosing process, it will cause increased spending on tourism activities [10], and they had to overcome this. So it is necessary to develop technology in assisting in recommending destination choices for tourists that 
in this study is referred to as a tourism destination recommendation system (TDRS).

In several studies on tourism recommendations, several researchers used the concept of a single criterion and multi-criteria combined with several methods, for example, based on Multi-Criteria Decision Making (MCDM), fuzzy logic, machine learning, and others [11-14]. TDRS in this study uses the concept of machine learning-based Multi-Criteria Recommendation System (MCRS). On that concept, recommendations are generated based on other visitor experience databases [15], for example, in terms of evaluating the characteristics of tourism destinations. Therefore we need the process of distributing data between parts in the TDRS. The three interrelated elements of TDRS in this study include users, servers, and sensors. Users are each traveler who assesses the characteristics of a tourism destination and sends it to the server. Users are also potential tourists who get the overall rating data of tourism destination characteristics from the server as a reference in generating recommendations. The server is a device equipped with machine learning to process tourism destination assessment data from the user so that the resulting characteristics of the tourism destination are sent to the user. While the sensor is a device that is tasked with collecting dynamic data related to tourist destinations such as popularity, traffic and weather to be sent to the user as a consideration in generating recommendations other than the weight of the characteristics of tourism destinations.

All parts in the TDRS are connected so that the process of data sharing between them can run well. Therefore we need a network architecture to be used as a means of distributed and secure data sharing data. There are two basic architectural models in the data sharing process, centralized and decentralized [16]. The centralized architecture has the characteristic of a centralized network so that each data transaction always passes through the central node. Centralized architecture has a risk of failure in the network, which is when a node in the middle is damaged. The use of this architecture can increase the time of execution of data transactions because each transaction must go through approval from the central node. Decentralized architecture has better capabilities compared to centralized architecture, where each node can communicate with other nodes without passing through the central node. This characteristic is certainly more suitable to be used as architecture in the process of sharing data between tourists, servers, and sensors in this TDRS.

One technology that is implementing a decentralized architecture and is starting to become a trend in research on data sharing is the blockchain. Besides having the ability to handle distributed data sharing, blockchain also uses decentralized networks with cryptographic techniques for data security [17] [18]. Blockchain referred to as a general-purpose data structure; this makes it very possible to use in application domains other than cryptocurrency.

This study uses blockchain as an architecture to improve the capability and security of the data sharing process between parts of the proposed system called Decentralized TDRS (DTDRS). In this work, the blockchain handles the multi-criteria rating data circulation required by MCRS as a reference in producing tourism destination recommendations for users. This decentralized architecture takes the tourism destination rating data from the user, machine learning data on the server node, and also data from the sensor node. Each blockchain-based data sharing function is built in a web platform to be accessible using a mobile device or computer. We tried to develop our data-sharing platform to make it easier and free to implement the design to the data characteristics and functions of the system.

\subsection{Relate work}

In 2019, paper [19] proposed a neural networkbased recommendation system using the concept of data sharing that can overcome data circulation for different systems. However, this study only uses one variation of data, namely information data for items. Rules and guidelines for developing data-sharing systems to support recommendation systems are discussed in a paper [20]. Through a paper [21], Frey proposes a blockchain-based recommendation system to improve data sharing security. The recommendation system in the paper uses a collaborative filtering approach. The characteristic of this approach is that it still uses the single rating criteria for items as reference data in generating recommendations.

Another study that proposed Xue carried out a blockchain-based data-sharing system in 2017 through a paper [22]. The data-sharing system on these papers used to address the exchange of medical data between institutions. The proposed system scheme can solve the problem of checking, saving, and synchronizing medical data by updating the consensus mechanism used. However, the proposed system is still not designed to interact with machine learning. In 2017 paper [23] proposed a blockchainbased framework to handle the data sharing process. This study takes advantage of blockchain technology, namely immutability and the built-in autonomy, to control access to sensitive data stored in the cloud. 
Furthermore, paper [24] proposes a blockchainbased medical record management system that can improve management and data sharing processes for electronic medical records. The system only manages between the two parties, namely patient and healthcare provider. The author of the paper [25] also proposes a data-sharing architecture and protection scheme for blockchain-based medical records. The author designed an architecture to handle data sharing between user devices, servers, and system managers. Papers [24] and [25] have not used and benefited from machine learning, sensors, or IoT in their proposed architectures.

Paper [26] proposes a decentralized system for managing blockchain-based personal data. In this paper, the authors designed a blockchain-based system to handle two types of non-financial transactions. Blockchain also monitors and controls access to personal data. The system sends an access transaction when a user tries to access the database for data retrieval and storage. Users explain the need for data transactions after transaction access is approved. The goal is to complete communication between nodes on the blockchain. The author of the paper focuses more on access control on the blockchain and has not considered the variation of the data used. The author in the paper [26] introduced a decentralized system for blockchain-based energy trading. Their discussion focuses on a secure transaction exchange system. Decentralized systems improve security problems, privacy problems, and scalability that usually arise in centralized systems. The discussion in this paper focuses on blockchain applications for smart medical systems. Paper [27] proposes a blockchain-based decentralized storage system and implements it in an air-to-ground industrial network. In this paper, blockchain handles data circulation between several types of nodes involving IoT technology in its architecture. One of the focuses of the author's discussion is the heterogeneous trading network based on blockchain technology.

In [28], the author proposes an architecture in blockchain-based vehicle network applications. The blockchain architecture is designed with security and distributed storage of big data in mind. The author uses blockchain to handle vehicle data transmission between nodes connected to the network. In the paper [29] the author uses blockchain to support shared systems in manufacturing. The author implements the etherium framework to build a prototype system. The architecture proposed in the paper divides the system into several parts, and the blockchain handles the process of exchanging data between them by utilizing a consensus mechanism, incentive mechanism, and smart contracts.

\subsection{Contributions and organization of paper}

Research on blockchain-based data-sharing systems, especially for recommendation systems, is exciting to explore because there are not many papers focused on it. The system that has been proposed, for example, in paper [21], still uses the single criteria concept. In comparison, the recommendation system with the multi-criteria concept has many advantages compared to single criteria. In contrast, the recommendation system with the multi-criteria concept has many advantages compared to single criteria. The recommendation system with the single criteria concept uses a more uncomplicated assessment reference for items, namely, one item has one rating criterion.

Meanwhile, the recommendation system with the multi-criteria concept has more detailed and more assessment references, resulting in more precise recommendations. However, this also requires the support of a more robust and secure data sharing system. The recommendation system for selecting tourist destinations has a variety of data criteria references, so it is more suitable to use the multicriteria concept. Several studies discussing data sharing systems, for example, in papers [22 - 24] and [25], are aimed at fields other than tourism recommendation systems that have different data characteristics, data sharing scenarios, and system architectures. Therefore, the motivation for this paper is to design a blockchain-based data-sharing system for the selection of tourism destinations with the concept of a multi-criteria recommendation system. Some of the contributions from this paper described as follows.

First, we propose a blockchain-based datasharing system to support data circulation on a multicriteria recommendation system for selecting tourist destinations. The data-sharing system handles the circulation of several types of data, including rating data on user ratings of items, predictive data overall ratings from machine learning, and dynamic data on popularity, traffic, and weather from IoT-based sensor nodes. In this study, the data-sharing system can interact with machine learning equipment and IoT-based equipment.

Second, we designed a decentralized recommendation system architecture to handle data communication between the three types of nodes connected in the network, namely user nodes, servers, and sensors, which are parts of the DTDRS. To make it easier to understand the data communication 
between them, we describe the reference steps for data communication between nodes.

The development, testing, and analysis of the DTDRS data sharing system are explained in several sections contained in this paper. Section 2 discusses preliminaries in choosing tourism destinations, recommendation systems, and blockchain. Section 3 discusses the proposed system, including data communication, network architecture, and block structure. Furthermore, the results of the experiment and its discussion are shown in section 4. Finally, we conclude the contents of this paper in section 5 .

\section{Preliminaries}

\subsection{Choosing tourism destinations}

Based on several studies, tourism destination has several definitions. In geographical terms, a tourism destination is an area chosen by tourists as a destination in tourism activities, including supporting facilities they have [30]. Tourism destinations can also interpret as places where there are tourism products and services that motivate tourists to visit and travel [31]. Everyone certainly has different motives when visiting a tourism destination, depending on their preferences and characteristics. The correct selection of tourism destinations will increase satisfaction and can maximize the traveling experience. So selecting tourism destinations is an essential process in a series of tourism trips.

In some studies, tourism activities divided into three main phases, namely before the trip, during the trip, and after the trip [1][7]. Each phase certainly has a different influence on tourist trip activities. Among the three phases, before the trip is the initial and primary phase, which contains activities related to preparation before traveling. The activity includes gathering information about the characteristics of the tourism destination choice followed by determining travel decisions, including preparing the travel such as booking travel tickets and hotel rooms. In determining the decision, there is a process of selecting tourism destinations, choosing the priority of places to visit according to the expectations, characteristics, and preferences of the tourists.

Juan L. Nicolau states that two factors influence the selection of tourism destinations; there are Personal Characteristics (PC) and Destination Attributes (DA). The PC variable describes the appearances and characteristics that influence the tourist's choice of destinations. These variables include motivations, income, age, gender, marital status, educations, and nationality. The DA variable shows the characteristics belonging to each destination, including attractions, prices, surface area, attributes of nature, infrastructure, accessibility, distance [32].

In other studies, researchers mentioned that they used the framework as an assessment of the facility and service in tourism destinations, namely 6As Tourism Destinations (6AsTD) [31, 33]. Each component of the framework represents the DA of a tourism destination. These components include attractions, accessibility, amenities, available packages, activities, and ancillary services [34]. In this paper, we use DA in the 6AsTD framework as a tourist reference for assessing the tourism destinations characteristics. The dynamic characteristic factors of tourism destinations also influence destination determination activities, for example, the level of popularity [35], traffic, and weather [36]. The dynamic characteristic is another consideration in determining tourism destinations. The system can make dynamic data retrieval using sensors by utilizing the internet of things (IoT) technology. In a study, IoT technology influences increasing the ability of a recommendation system[37].

\subsection{Recommendation system}

Recommendation system (RS) is one of the branches in the field of information retrieval and artificial intelligence. RS is a software that functions to predict item choices based on user characteristics [38]. The purpose of the RS is to develop a mathematical model or objective function that can predict how likely users are to like an item [39]. In this study, the users are tourists, and the items are tourism destinations. RS is part of Decision Support Systems (DSSs), which are systems that can recommend items based on user characteristics and preferences [40]. In the field of tourism, user characteristics are the user characteristics assessment for tourism destinations.

Much research on RS uses a single ranking as a ranking system to represent user preferences for items. The rating can be in the form of numerical, binary, and so on. However, some research works consider the use of a single rank as a limitation on RS because a single rating cannot represent various characteristics of items [41]. The suitability of the items recommended for the user may depend on several criteria for consideration in determining the choice. But the use of several criteria makes it difficult for users to choose the best alternative from the available options. Therefore, this study proposes a multi-criteria recommendation technique as a solution to the problem by using several ratings for 
some essential characteristics of the items to improve the accuracy of system predictions.

MCRS uses different attributes of items to describe the quality of an item. For example, a recommendation system about music, criteria, or attributes might be lyrics, visuals, audio, sound, beat, genre, etc. The multi-criteria approach enhances the traditional approach by considering various attributes of items that might be liked by the user. Through MCRS, users can rank their subjective preferences on some of the attributes that an item has [42].

An RS can use the additional information provided by a multi-criteria rating to provide more accurate recommendations to its users. MCRS adds to the ability of RS by considering ratings as multi values, not one value. Eq. (1) shows a utility-based formula in a multi-criteria recommendation. In the MCRS, the system assigns ratings for items based on several parameters. Multi-criteria rating systems can choose the user utility model for items given with an overall rating of $R_{0}$ as well as user $R_{l}, \ldots, R_{k}$ for each criterion c $(c=1, \ldots, k)$. Some systems can choose not to use the overall rating and only focus on individual rating criteria.

$$
R: \text { User } \times \text { Item } \rightarrow R_{0} \times R_{1} \times \cdots \times R_{k}
$$

MCRS uses several ratings assigned to several essential characteristics of items to improve the accuracy of system predictions. Along with the development of RS, machine learning is one of the techniques used to model assessment criteria within the framework of the final assessment of users based on their responses to the assessment criteria [43]. In other studies, some machine learning algorithms used for MCRS modeling, for example, neural networks [44] or K-means [45][46]. In a study, Neural Network as a machine learning method is used to study the relationship between $\mathrm{R}_{0}$ and $\mathrm{R}_{\mathrm{k}}$ for $\mathrm{k}=1,2, \ldots ., \mathrm{n}$ as shown in Eq. (2) [41].

$$
R_{0}=f\left(R_{1}, R_{2}, R_{3}, \ldots, R_{n}\right)
$$

The purpose of using MCRS machine-based learning in this study is to improve RS capabilities. The assessment of DA $\left(R_{1}, R_{2}, \ldots, R_{n}\right)$ conducted by tourists becomes the primary data processing by machine learning to produce the $\mathrm{R} 0$ value needed in the recommendation system calculation.

\subsection{Blockchain-based data sharing}

There are two standard data-sharing network architecture models discussed in many studies, namely centralized and decentralized, as shown in

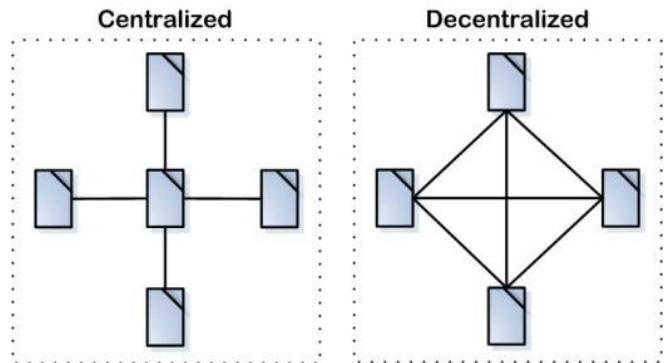

Figure. 1 The centralized and decentralized network

Fig. 1 [16]. Centralized is the traditional model, which is an architectural model where each node must communicate and get approval from the central node before communicating with other nodes. Decentralized is an architectural model that is currently popular and can also be called a peer to peer network [47]. Each node can communicate with all nodes that connected without having to go through the approval of the middle node. The process certainly facilitates communication between nodes easier. By using a decentralized architecture model, each data distributed to every node connected in the network without exception. Decentralized architecture is suitable to be applied in TDRS given the number of possible nodes to be connected in the network.

Blockchain is a decentralized distributed data sharing architecture model used in this study and first introduced by Satoshi Nakamoto through the Cryptocurrency application called Bitcoin. Each block in the blockchain has several parts, namely Block Number, Previous Hash, Current Hash, Nonce, Data, and Timestamp [48]. Blockchain is not only used in the cryptocurrency field but also developed to support the process of sharing data in other areas such as medical records [25], RFID system [49] or intelligent vehicles [28]. In another study, researchers successfully implemented a blockchain to handle data sharing on a tourism destinations rating system [50]. In this study, we propose a blockchain to support data sharing between nodes in a tourism destination recommendation system.

Blockchain has several characteristics, which are immutability, decentralization, anonymity, increased capacity, and better security [48]. Blockchain offers solutions to overcome the problem of communication and verification of mobile device transactions [51]. Data security is guaranteed based on the hash (previous hash and current hash) as a numeric digest of data used to verify the transaction data. SHA256 is a standard algorithm used to get hash values on blockchain [52]. Through this algorithm, the data in a block converted into random characters as a representation of the hash value used to build a block. 
After obtaining the hash value through the algorithm, then one of the next essential processes in the blockchain is the chaining block. Chaining block is a process where each block is chained together with other blocks through the hash value of the previous block. Finally, each block is chained and used in the transaction process between nodes.

Each node that connected in a blockchain network can conduct data transactions directly with other nodes. There is no centralized part in charge of verifying the blockchain validity, and a mechanism is needed to reach consensus agreement in the network. In some studies, the mechanism is an algorithm called Proof of Work (PoW), Proof of Stake (PoS), or Proof of Activity (PoA) [53, 47]. PoW is the most popular algorithm and used in Bitcoin and Etherium. In the PoW algorithm, a node publishes the next block by being the first to solve the computational puzzle. This algorithm uses a challenging puzzle to solve, but it is easy to check its validity. All nodes can quickly validate each proposed block, and the node can reject the block if it is invalid [16]. Within the blockchain, it is possible to verify transactions without analyzing all nodes in the network. A user only needs to save a copy of the most extended block header from PoW. The user requests a copy to the network node until he ensures that it has the longest chain. Even though the user cannot check the transaction for himself, but by connecting to a place in the chain, he can find out that the network node has received it. Then a block can be added after further confirming that the network has accepted it.

\section{Blockchain design for DTDRS}

\subsection{Data communication}

Never The DTDRS proposed in this study uses a decentralized network that has three primary nodes, namely users, servers, and sensors. The Blockchain network connects each node to support the process of decentralized data sharing in this study. Although they are interconnected, each node has a varying direction of communication. The user node sends data on the assessment of tourist destination characteristics to the server according to the 6AsTD framework. The data consists of an assessment of the attractions component $\boldsymbol{R}_{\mathbf{1}}$, accessibility $\boldsymbol{R}_{\mathbf{2}}$, amenities $\boldsymbol{R}_{\mathbf{3}}$, available packages $\boldsymbol{R}_{\mathbf{4}}$, activities $\boldsymbol{R}_{\mathbf{5}}$ and ancillary services $\boldsymbol{R}_{\mathbf{6}}$. The collection of assessment data is processed by machine learningbased MCRS to produce recommendations for tourist destination choices for users. The series of processes is not only done in user devices but also in server devices, especially to handle the process of machine learning. This division of operation has the purpose of the complexity of the process in which machine learning does not burden users. Following Eq. 2, the value of $\boldsymbol{R}_{\mathbf{0}}$ generated by the server is sent to the user to complete the value needed in the calculation of the recommendation system on the user's node device.

In this study, recommendations generated from the MCRS process compared to data on the dynamic conditions of the travel destination. The goal is to get recommendations that are not only following user expectations but also following the dynamic data conditions of tourist destinations. The sensor node sends each data obtained in realtime to the user node. The sensor node is a computer device that can read dynamic data that is owned by a tourist destination. The device retrieves data over the internet, then classifies it to the user as dynamic data. The data sent to user nodes include popularity $\boldsymbol{D} \boldsymbol{y}_{\mathbf{1}}$, traffic $\boldsymbol{D} \boldsymbol{y}_{\mathbf{2}}$ and weather $\boldsymbol{D} \boldsymbol{y}_{3}$. All data sent by the three types of nodes in the network shown in Table 1.

The three types of nodes have variations and treatments for data, causing the data communication process between them to be different. Table 2 shows the steps of data communications between nodes in the DTDRS.

\subsection{Network architecture and block structure}

Each node in the DTDRS network architecture can communicate with each other. Fig. 2 shows the relationship between nodes in the blockchain network architecture to handle the distribution of data between them. Each driver connected to the network through an application on their mobile device called a user node. Users connected in a network can communicate with all nodes, whether other users, servers, or sensors. Users do two-way data communication with the server and other users, but can only transmit data one-way with sensors. The server has a communication relationship with the user, but it is not related to the sensor. In the blockchain network architecture in DTDRS, the sensor is authorized only to send data to users.

The first step done in designing a blockchain network architecture is to define the block structure used. Nodes transmit each record in the block form.

Table 1. Data sent by each node

\begin{tabular}{|l|l|}
\hline Direction & Data \\
\hline User to Server & $R_{1}, R_{2}, R_{3}, R_{4}, R_{5}, R_{6}$ \\
\hline User to User & $R_{1}, R_{2}, R_{3}, R_{4}, R_{5}, R_{6}$ \\
\hline Server to User & $R_{0}$ \\
\hline Sensor to User & $D y_{1}, D y_{2}, D y_{3}$ \\
\hline
\end{tabular}


Table 2. Steps of data communication between nodes

\begin{tabular}{|c|c|c|c|}
\hline Nodes & Process & Sequence of Steps & Information \\
\hline \multirow[t]{2}{*}{ User } & $\begin{array}{c}\text { Sending of tourism } \\
\text { destinations rating } \\
\text { data }\end{array}$ & $\begin{array}{l}\text { 1. Users enter tourist destination assessment } \\
\text { data }\left(R_{1}, R_{2}, R_{3}, R_{4}, R_{5}, R_{6}\right) \\
\text { 2. Enter } R_{1}, R_{2}, R_{3}, R_{4}, R_{5} \text {, and } R_{6} \text { into the } \\
\text { tourism destinations assessment database } \\
\text { 3. Enter } R_{1}, R_{2}, R_{3}, R_{4}, R_{5} \text {, and } R_{6} \text { into the } \\
\text { block } \\
\text { 4. The process of Hashing, chaining, and } \\
\text { broadcast blocks }\end{array}$ & $\begin{array}{c}\text { The } \\
\text { destinations } \\
\text { nodes are } \\
\text { Users and } \\
\text { Server }\end{array}$ \\
\hline & Receiving data & $\begin{array}{l}\text { 1. Get data from block } \\
\text { 2. Reads origin node based on NodeID } \\
\text { 3. If origin node = User, then enter data into } \\
\text { the tourism destinations assessment } \\
\text { database } \\
\text { If origin node = Server, then enter data into } \\
\text { the tourism destinations assessment } \\
\text { database } \\
\text { If origin node = Sensor, then enter data into } \\
\text { the database of dynamic data }\end{array}$ & $\begin{array}{l}\text { The origin } \\
\text { nodes are } \\
\text { Users, Server, } \\
\text { and Sensor }\end{array}$ \\
\hline Server & $\begin{array}{l}\text { Sending and } \\
\text { receiving of data }\end{array}$ & $\begin{array}{l}\text { 1. Get data from block } \\
\text { 2. Reads origin node based on NodeID } \\
\text { 3. If origin node = User, then data = tourist } \\
\text { destination assessment data }\left(R_{1}, R_{2}, R_{3}, R_{4} \text {, }\right. \\
\left.R_{5}, R_{6}\right) \\
\text { 4. Send tourist destination assessment data to } \\
\text { the server database } \\
\text { 5. Get data from the server database } \\
\text { 6. Processing the data using machine learning } \\
\text { to produce the value of } R_{0} \\
\text { 7. Enter } R_{0} \text { into the block } \\
\text { 8. The process of hashing, chaining, and } \\
\text { broadcast blocks }\end{array}$ & $\begin{array}{c}\text { The origin and } \\
\text { destination } \\
\text { node are } \\
\text { Sensors }\end{array}$ \\
\hline Sensor & Sending data & $\begin{array}{l}\text { 1. Perform dynamic data }\left(D y_{1}, D y_{2}, D y_{3}\right) \\
\text { retrieval processes and save them into the } \\
\text { database } \\
\text { 2. Get } D y_{1}, D y_{2}, D y_{3} \text { from the database of } \\
\text { dynamic data } \\
\text { 3. Enter } D y_{1}, D y_{2}, D y_{3} \text { into the block } \\
\text { 4. The process of Hashing, chaining, and } \\
\text { broadcast blocks }\end{array}$ & $\begin{array}{c}\text { The } \\
\text { destinations } \\
\text { nodes are } \\
\text { Users }\end{array}$ \\
\hline
\end{tabular}

Part of the block structure adapted to the needs of data blocks used to produce recommendations of tourism destinations. Fig. 3 shows that the study has three parts of the block structure, which are Block Information (BI), Hash, and Data.

$\mathrm{BI}$ is part of a block that consists of three parts called block number $b n$, nonce $n n$ and time stamp $t s$. Block number contains a sequence of numbers that represent the identity of a block so that each block in the blockchain has a block number that is different from others. Nonce, as the second part, contains a combination of random numbers that characterizes each block. A nonce is an essential part because it is used in the process of finding blocks when they are in a blockchain series. The timestamp is a part that contains information about the date and time combination of block-creation.

Data $D$ in the block structure contains nodeID id and sent by node data $\mathrm{s} d n$. How to set the two data sections separated using the \# character as follows.

$$
D=i d \# d n
$$




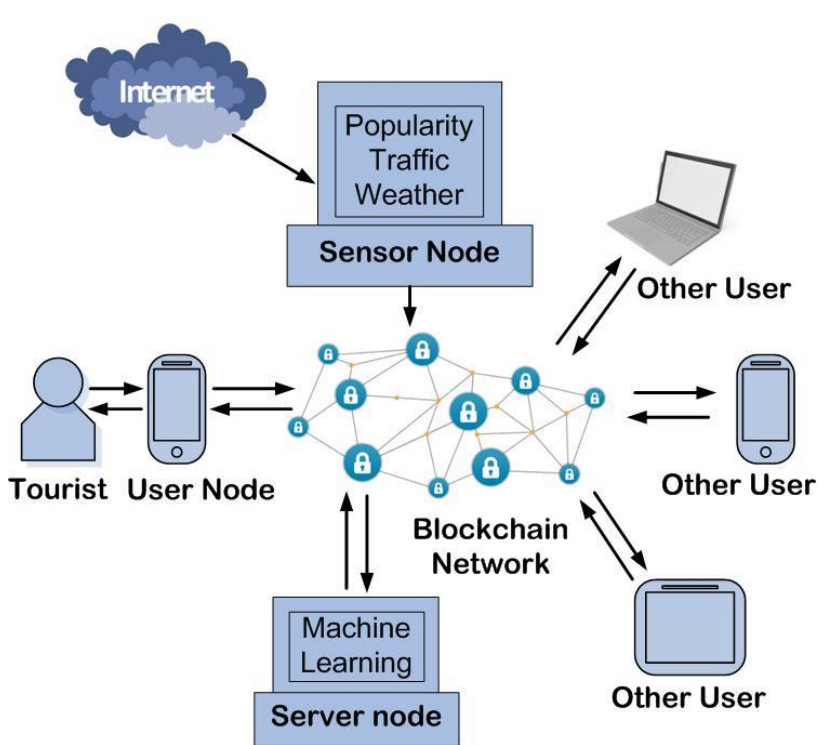

Figure. 2 The network architecture of DTDRS

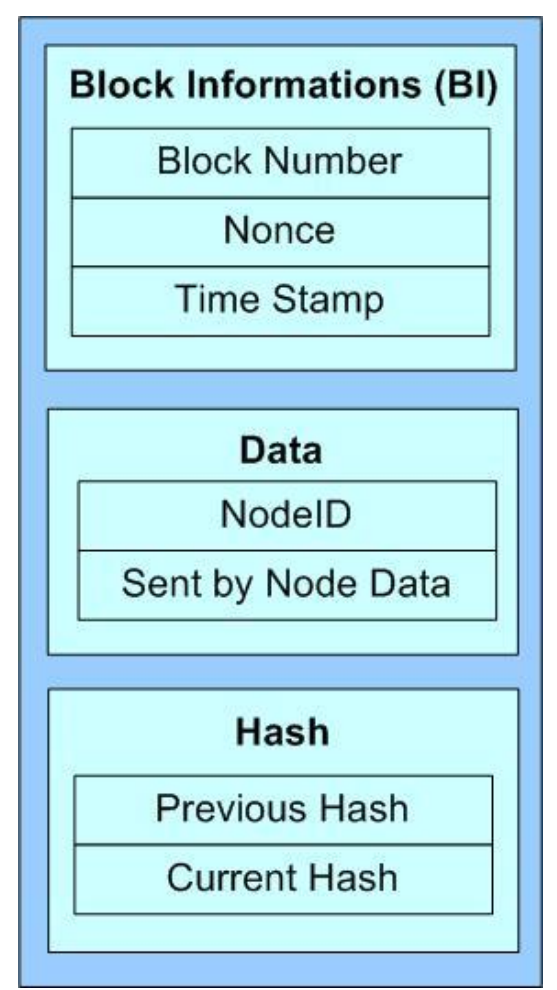

Figure. 3 Block structure figure

NodeID is a unique identity that is owned by each node. Sent by node data is the data that each block will carry through the blockchain network. The data varies according to the sending node, as shown in table 1. For example, if the block structure has data sent by the user node, it contains $R_{1}, R_{2}, R_{3}, R_{4}, R_{5}$, and $R_{6}$. If the block structure has data transmitted by the server node, it contains $R_{0}$. But if the block structure has data sent by the sensor node, it includes $D y_{1}, D y_{2}$, and $D y_{3}$. The configuration data transmitted by the user node is as follows:

$$
D=i d \# R_{1} \# R_{2} \# R_{3} \# R_{4} \# R_{5} \# R_{6}
$$

The configuration data sent by the server node is as follows:

$$
D=i d \# R_{0}
$$

While the configuration of data sent by the sensor node is as follows:

$$
D=i d \# D y_{1} \# D y_{2} \# D y_{3}
$$

The hash $H$ in the block structure designed consists of numeric digest generated through the hashing algorithm, which is often used also in other blockchain applications, namely SHA256. There are two types of hashes in this section, previous hash $p h$, and current hash $c h$. The previous hash is the hash value obtained from the hash in the previous block. The current hash itself contains values generated from the process of hashing data in a block. The hash value is obtained based on the function of Eq. 3 .

$$
H=f(b n+p h+t s+D+c h+n n)
$$

The hashing block process uses validation of the hash value with proof of the work done to get the hash value according to the specified target. In the validation process, the system checks for the hash value generated through the hashing process as using the following equation.

$$
H(n n\|p h\| t s\|D\| \ldots)<T
$$

In Eq. 4, the variable $T$ is the predetermined hash target value. The system successfully validates the hash value of the block when the hash value generated meets the specified target. The stages of checking the validity of a block using proof of work are as follows.

Step 1: $\quad$ Preparing block structure data, $p h+$ $t s+D+c h+n n$.

Step 2: $\quad$ Runs hashing function on data $p h+$ $t s+D+c h+n n$.

Step 3: If the initial value of the hash is not the same as the target value, then increment $n n$ and repeated to step 2 .

Step 4: If the initial value of the hash is the same as the target value, then the proof of work is achieved.

\subsection{Chain of the block}

After the hashing process, the system performs a broadcast to create a chain of the block. Previously, 


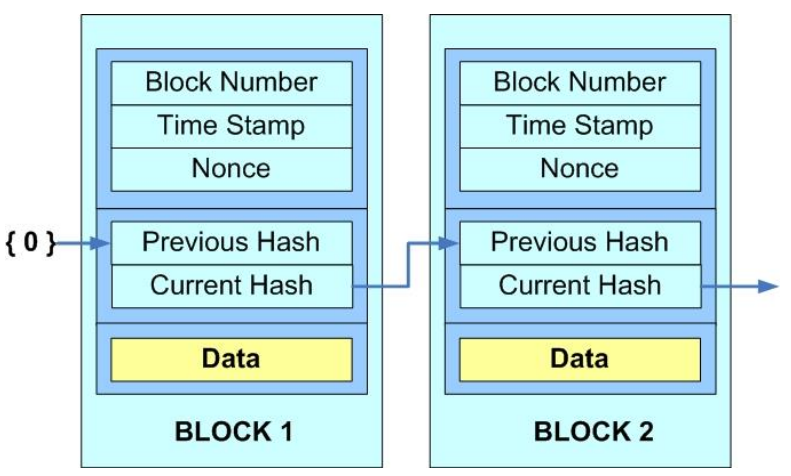

Figure. 4 Chain of block

the blockchain system checked the block to determine where in the chain a block placed itself. Each added block will then create a chain of the block on each interconnected node. The system adds blocks that have been validated by proof of work into the blockchain. Then checked the positioning of the placement block to the blockchain. The system has a chain position for the validated block before the block occupies a place on the blockchain. The system adds blocks that have been validated by proof of work into the blockchain. Then checked the positioning of the placement block to the blockchain. The system performs a broadcast block to the blockchain network after checking the location of the validated block placement successfully.

Each block is linked based on the previous hash value obtained from the current hash value in the last block before. So if the data of the last block changes, it will change the hash value too. If the hash value changes, then this results in the next block, also having a different hash value because the formation of a block is also the result of the previous block hash value. By doing that, the blockchain will be able to quickly detect or even reject if an unwanted block changes occur to secure the transaction process in the blockchain.

Fig. 4 shows that the first block is a unique block, which is the initial block where there is no other block before the block. So the first block has a previous value that is not a representation of the last block before. Therefore the previous hash value in the first block is 0 , as an indicator that this is the first block. Whereas the second, third, and so on blocks have previous hash values from the current hash values in the last block before. For example, the second previous block is the current block of the first block, and the third previous block is the current block of the second block, and so on. Each block creates a linked blockchain after it has succeeded in the broadcast. Blocks containing data from each node in the network are interconnected and create a blockchain ledger record that will be visualizing to the table form.

\section{Result and discussion}

\subsection{Experimental}

There are three parts of the data-sharing system for DTDRS in this study, namely the user, server, and sensor. At the user node, the system uses a web platform to build a data-sharing system. The aim is to make it more flexible to use on various technology platforms, from mobile devices to computers.

Fig. 5 shows an example of the user interface in the process of sending tourism destination assessment data available at the user node. In the experimental phase, we build machine learning on server nodes to process data tourism destinations assessment from users. Through the blockchain network, the user node sends $R_{1}, R_{2}, R_{3}, R_{4}, R_{5}, R_{6}$ to the server node, and the server node sends $R_{0}$ to the user node. Fig. 6 shows an example of receiving the data between the server and the user device.

In this study, we use a web platform to build systems on sensor nodes. Users get dynamic data on

\section{Tourism Destination Assessment}

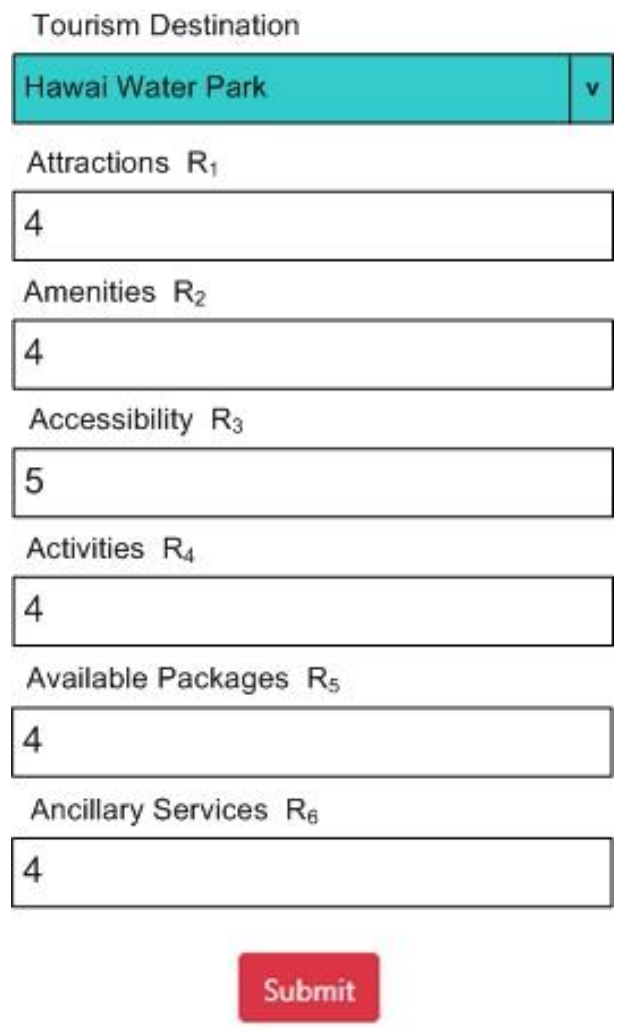

Figure. 5 The user interface of tourism destinations assessment 


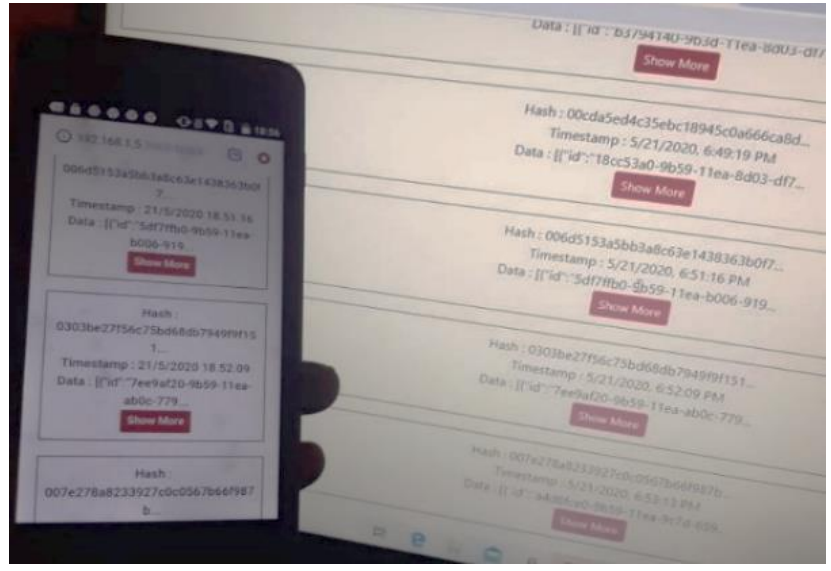

Figure. 6 The example of sending data from server to user

\begin{tabular}{|c|ccc|}
\hline \multicolumn{3}{|c|}{$\begin{array}{l}\text { Thusday, March 26, } 2020 \\
\text { 11:2:1 }\end{array}$} \\
\hline Data Export \\
\hline Malang Tourism Destinations Popularity Rating \\
\hline $\begin{array}{c}\text { Popularity } \\
\text { Rating }\end{array}$ & $\begin{array}{c}\text { Destination } \\
\text { Name }\end{array}$ & $\begin{array}{c}\text { Tourism } \\
\text { Type }\end{array}$ & $\begin{array}{c}\text { Positive } \\
\text { Score }\end{array}$ \\
\hline 1 & Hawai Water Park & Artificial TIrta & 5.38 \\
\hline 2 & Bromo & Adventure & 3.88 \\
\hline 3 & Coban Sewu & Waterfall & 2.89 \\
\hline 4 & Candi Kidal & Historical & 2.24 \\
\hline 5 & Candi Badut & Historical & 1.72 \\
\hline 6 & Teluk Asmara & Beach & 1 \\
\hline 7 & Coban Pelangi & Waterfall & 0.99 \\
\hline 8 & Budug Asu & Adventure & 0.94 \\
\hline
\end{tabular}

Figure. 7 Dynamic data retrieval of popularity

tourism destinations from the sensor node through the web platform. Fig. 7 shows an example of dynamic data retrieval of popularity. To get data on the popularity of tourist destinations, we use a reference number of tweets on Twitter.com. In addition to popularity, sensor nodes provide other data, namely weather, and traffic. Sensor nodes get weather data through the weather Application Programming Interface (API) provider site. Meanwhile, to get traffic data, we utilize Google Map service. Furthermore, the sensor node sends dynamic data that is ready for the user through a blockchain network.

\subsection{Blockchain result}

We conducted several stages of testing to determine the performance of the DTDRS system design. The first step is to test the hashing process, where the $b n+p h+t s+D+c h+n n$ values entered in the hashing function are successfully converted to hash values. Fig. 8 shows an example of

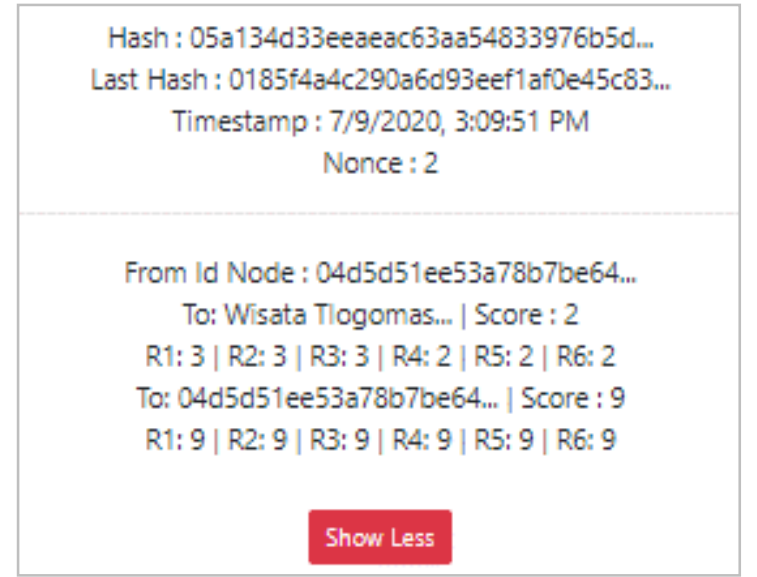

Figure. 8 An example result of the hashing process

From ch

of Block 1

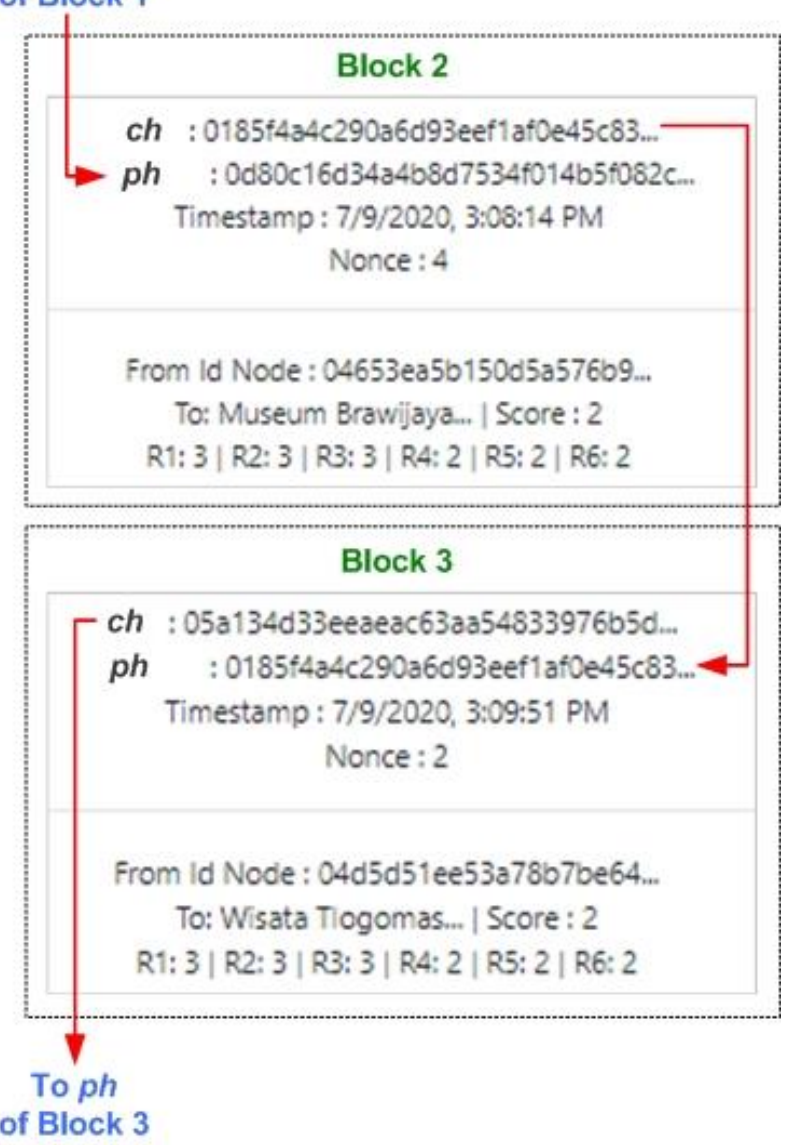

Figure. 9 An example result of the chaining result

the hashing process results in assessing tourism destination data. The result of the hashing process contains hash data, timestamp, nonce, nodeID, and the values of $R_{l}$ to $R_{6}$.

After testing the hashing process, the next step is to check the results of the chaining block and proof of work processes. We do peer to peer ( $22 p)$ testing in the communication scheme between several nodes connected in a blockchain network. Fig. 9 shows the results where each address node sends an assessment 
Table 3. Example of hashing and mining result on server node in $\mathrm{p} 2 \mathrm{p}$ network

\begin{tabular}{|c|c|c|c|}
\hline $\begin{array}{c}\text { Num } \\
\text { ber }\end{array}$ & $\begin{array}{c}\boldsymbol{R} \boldsymbol{0} \\
\text { Score }\end{array}$ & $\begin{array}{c}\text { Time of } \\
\text { Mine } \\
(\mathbf{m s})\end{array}$ \\
\hline 1 & 3,33 & 00700919ce994ab626a & 368.5 \\
\hline 2 & 4,16 & 01ba447324cbd99afb5 & 39.1 \\
\hline 3 & 3,16 & 00985ace5d3928d9d3a & 427 \\
\hline 4 & 4,33 & 03fa3ec5d89457cedd51 & 25.9 \\
\hline 5 & 3,5 & 01b49eaea3afaba815a & 34.4 \\
\hline 6 & 2,83 & 0d29eb853ece2c60d2c & 17.3 \\
\hline 7 & 3,16 & 2df7d0c0d49569a0a23 & 31.4 \\
\hline 8 & 3,5 & 256fffb4b6372edc 4958 & 9.2 \\
\hline
\end{tabular}

of a tourist destination through internet browser access. From the results of data sharing, the system successfully formed a chain of the block to connect between blocks. The value of $p h$ and $c h$ in each block becomes a link between the blockchain. Block 2 uses the same $p h$ value as $c h$ in block 1. Also, block 3 has the same $p h$ value as the value of $c h$ owned by block 2 .

Proof of work performance testing aims to find out whether each hash value of each block meets the set proof of work target. The intended proof of work target is that the resulting hash value must begin with a zero digit. Table 3 shows the results of the hashing and mining process in the $\mathrm{p} 2 \mathrm{p}$ network of sending machine learning result data $R_{0}$ from the server to the user. Of the eight examples of hash results, numbers $1,2,3,4,5$, and 6 are manifestations of hash values that meet the target proof of work, namely the prefix hash zero digit value. While numbers 7 and 8 are examples of hash values that do not meet the target proof of work where the initial hash digit starts with a non-zero number. The difference in the hash value becomes a reference in proof of work to determine the validity of a block. Proof of work is an essential process because only valid blocks are added to the chain-block. The table also shows that the fastest time of mine is $9.2 \mathrm{~ms}$, with an average of $119.1 \mathrm{~ms}$.

Table 4 shows the results of the hashing and mining process of sending multi-criteria rating $R_{1}, R_{2}$, $R_{3}, R_{4}, R_{5}, R_{6}$ from user to server, and user nodes in the $\mathrm{p} 2 \mathrm{p}$ network. In the table, the fastest time of mine is $15.4 \mathrm{~ms}$ from the values data of multi-criteria rating $3,3,3,2,2,2$. Meanwhile, the average time of mine from all examples of hashing and mining results is $85.4 \mathrm{~ms}$.
Table 4. Example of hashing and mining result on user node in $\mathrm{p} 2 \mathrm{p}$ network

\begin{tabular}{|c|c|c|c|}
\hline $\begin{array}{c}\text { Num } \\
\text { ber }\end{array}$ & $\begin{array}{c}\boldsymbol{R}_{\mathbf{1}}, \boldsymbol{R}_{\mathbf{2}}, \\
\boldsymbol{R}_{\mathbf{3}}, \boldsymbol{R}_{\mathbf{4}}, \\
\boldsymbol{R}_{\mathbf{5}}, \boldsymbol{R}_{\mathbf{6}} \\
\text { Scores }\end{array}$ & Hash Value & $\begin{array}{c}\text { Time of } \\
\text { Mine } \\
(\mathbf{m s})\end{array}$ \\
\hline 1 & $\begin{array}{c}3,3,3,2, \\
2,2\end{array}$ & $\begin{array}{c}\text { 0090a6576547a49c48f } \\
557828035 \mathrm{fd}\end{array}$ & 253.1 \\
\hline 2 & $\begin{array}{c}4,4,5,4, \\
4,4\end{array}$ & $\begin{array}{c}\text { 00b9719160689af4c2c } \\
187 \mathrm{~b} 27 \mathrm{fe} 891\end{array}$ & 90.9 \\
\hline 3 & $\begin{array}{c}4,5,5,4, \\
4,4\end{array}$ & $\begin{array}{c}\text { 005df5c1102d987cc5df } \\
25 \mathrm{c} 61 \mathrm{ec} 2 \mathrm{a} 1\end{array}$ & 41.2 \\
\hline 4 & $\begin{array}{c}3,2,4,3, \\
2,2\end{array}$ & $\begin{array}{c}\text { 0794e756aa791daa7f45 } \\
\text { b43f4b91d7 }\end{array}$ & 159,2 \\
\hline 5 & $\begin{array}{c}3,3,4,3, \\
3,3\end{array}$ & $\begin{array}{c}\text { 0d80c16d34a4b8d7534 } \\
\text { f014b5f082c }\end{array}$ & 81.6 \\
\hline 6 & $\begin{array}{c}3,3,3,2, \\
2,2\end{array}$ & $\begin{array}{c}\text { 0185f4a4c290a6d93eef } \\
1 \mathrm{af0e} 45 \mathrm{c} 83\end{array}$ & 15.4 \\
\hline 7 & $\begin{array}{c}4,4,4,3, \\
\text { a22da55cff23cf814bbe } \\
\text { 36945f59bb }\end{array}$ & 22.3 \\
\hline 8 & $\begin{array}{c}4,3,4,3, \\
3,3\end{array}$ & $\begin{array}{c}\text { 57c00e3cbcaa38b1b3e } \\
\text { 6d2553496d0 }\end{array}$ & 19.6 \\
\hline
\end{tabular}

\subsection{Comparison}

This research resulted in a data-sharing system to overcome several forms of data distribution from users, servers, and sensors. This study has different characteristics when compared to some ways of implementation of the data sharing blockchain in other studies. Table 5 shows these differences one by one. Where, reference [21] is research in the field of tourism, which focuses on the implementation of blockchain in recommendation systems to handle data sharing between users. References [25] is a research in the medical field, a data-sharing system built to overcome the circulation of data sources from user nodes and servers. The type of server used is a server without machine learning (non ML). References [29] is research that works in the field of manufacturing and has data sources from user nodes and sensors as a form of implementation of IoT technology. References [28] shows a study with more complete source data than the previous two studies. The study has data sources from users, servers, and sensors. The server in this study is a non ML type. If compared with the three studies, this study has the characteristics of completeness of the data source that is almost the same as research on references [28]. The difference is that this researcher has a data source from a server that has machine learning. Data sent from the server is the result of the machine learning process, which is data $R_{0}$. Each reference has a different type of data used. The use of multi-data has its difficulties compared to single data because the variation of data makes the process in the system more complex. 
Table 5. Blockchain data sharing comparison

\begin{tabular}{|c|c|c|c|c|c|}
\hline \multirow{2}{*}{$\begin{array}{c}\text { Refer } \\
\text { ences }\end{array}$} & \multirow{2}{*}{ Field } & \multicolumn{3}{|c|}{ Data Source } & \multirow{2}{*}{$\begin{array}{c}\text { Data } \\
\text { Used }\end{array}$} \\
\cline { 3 - 5 } & $\begin{array}{c}\text { Use } \\
\text { r }\end{array}$ & $\begin{array}{c}\text { Ser } \\
\text { ver }\end{array}$ & $\begin{array}{c}\text { IoT/ } \\
\text { Sensor }\end{array}$ & \\
\hline$[21]$ & Tourism & $\sqrt{ }$ & - & - & $\begin{array}{c}\text { Single } \\
\text { criteria } \\
\text { rating }\end{array}$ \\
\hline$[25]$ & Medical & $\sqrt{ }$ & $\begin{array}{c}\text { Non } \\
\text { ML }\end{array}$ & - & $\begin{array}{c}\text { Single } \\
\text { medical } \\
\text { data for } \\
\text { patient }\end{array}$ \\
\hline$[29]$ & $\begin{array}{c}\text { Manufact } \\
\text { ure }\end{array}$ & $\sqrt{ }$ & $\begin{array}{c}\text { Non } \\
\text { ML }\end{array}$ & $\sqrt{ }$ & $\begin{array}{c}\text { Multi } \\
\text { data of } \\
\text { manufact } \\
\text { ure }\end{array}$ \\
\hline$[28]$ & $\begin{array}{c}\text { Intelligen } \\
\text { t vehicles }\end{array}$ & $\sqrt{ }$ & $\begin{array}{c}\text { Non } \\
\text { ML }\end{array}$ & $\sqrt{ }$ & $\begin{array}{c}\text { Multi } \\
\text { data of } \\
\text { IoV }\end{array}$ \\
\hline Ours & Tourism & $\sqrt{ }$ & ML & $\sqrt{ }$ & $\begin{array}{c}\text { Multi- } \\
\text { criteria } \\
\text { rating }\end{array}$ \\
\hline
\end{tabular}

\section{Conclusions}

In this paper, we propose a blockchain-based data-sharing system to address data circulation between nodes in a tourism destination recommendation system with a detached node. Each node has a different direction of communication to make the data distribution needed to generate recommendations. The node user sends multi-criteria rating tourism destination data $R_{1}, R_{2}, R_{3}, R_{4}, R_{5}, R_{6}$ to the server, and other user nodes. Instead, the server node sends data from machine learning processing $R_{0}$ to the user nodes. The sensor node has a one-way communication direction, which only sends $D y_{1}, D y_{2}$, $D y_{3}$ data to the user node.

In the experimental stage, we built a system with a web-based platform. Every node that is part of this system sends data through the concept of a blockchain network that is decentralized. The results of experiments show that the data-sharing system based on blockchain technology work to overcome data distribution between nodes in DTDRS properly. The experiment results of sending $R_{0}$ data from the server node show that the average time of mine is $191.1 \mathrm{~ms}$, with the fastest time of mine is $9.2 \mathrm{~ms}$. Meanwhile, in the multi-criteria assessment data delivery of $R_{1}, R_{2}, R_{3}, R_{4}, R_{5}, R_{6}$ from user to the server, and other user nodes, the average time of mine is $85.4 \mathrm{~ms}$, and the fastest time of mine is $15.4 \mathrm{~ms}$.

Compared to previous related research, the system in this study has the advantage of being able to handle data circulation from a wider variety of nodes, including users, machine learning servers, and sensors. Besides, the system is also capable of handling multi-criteria assessment data circulation, such as data sent from user to server and other users.

\section{Conflicts of Interest}

The authors declare no conflict of interest.

\section{Author Contributions}

Conceptualization, Y.M. Arif, and M. Hariadi; methodology, Y.M. Arif, S.M.S. Nugroho, and M. Hariadi; software, Y.M. Arif, and H. Nurhayati; validation, Y.M. Arif, S.M.S. Nugroho, and M. Hariadi; formal analysis, Y.M. Arif, F. Kurniawan, S.M.S. Nugroho, and M. Hariadi; investigation, Y.M. Arif, S.M.S. Nugroho, and M. Hariadi; resources, Y.M. Arif, and H. Nurhayati; data curation, Y.M. Arif; writing - original draft preparation, Y.M. Arif, H. Nurhayati, and M. Hariadi; writing - review and editing, Y.M. Arif, F. Kurniawan, S.M.S. Nugroho, and M. Hariadi; visualization, Y.M. Arif, and H. Nurhayati; supervision, S.M.S. Nugroho, and M. Hariadi; project administration, Y.M. Arif, and H. Nurhayati; funding acquisition, Y.M. Arif, and H. Nurhayati.

\section{Acknowledgments}

Researchers completed this research in two laboratories, namely in Telematic Engineering Laboratory of Institut Teknologi Sepuluh Nopember Surabaya and Multimedia Laboratory of the Universitas Islam Negeri Maulana Malik Ibrahim Malang.

\section{References}

[1] U. Gretzel, M. Sigala, Z. Xiang, and C. Koo, "Smart tourism: foundations and developments", Electron. Mark., Vol. 25, No. 3, pp. 179-188, 2015.

[2] L. Etaati and D. Sundaram, "Adaptive tourist recommendation system: conceptual frameworks and implementations", Vietnam J. Comput. Sci., Vol. 2, No. 2, pp. 95-107, 2015.

[3] L. Kzaz, "Tourism Recommender Systems : An Overview of Recommendation Approaches", Int. J. Comput. Appl., Vol. 180, No. 20, pp. 9-13, 2018.

[4] P. K. Jithin, M. Vishnuram, P. Prasath, and J. T. Thirukrishna, "Tourism Guide for Tamilnadu ( Android Application )", Int. J. Innov. Res. Sci. Technol., Vol. 4, No. 11, pp. 112-116, 2018.

[5] D. Gavalas and M. Kenteris, "A web-based pervasive recommendation system for mobile tourist guides", Pers. Ubiquitous Comput., Vol. 15, No. 7, pp. 759-770, 2011. 
[6] F. B. Putra Prakasa, M. Radja, and Suyoto, "User experience based mobile application design for boat loaning at Marine Tourism in Indonesia", Int. J. Interact. Mob. Technol., Vol. 14, No. 4, pp. 86-102, 2020.

[7] N. A. Dwityas, "Social Media in Travel Decision Making Process", Int. J. Humanit. Soc. Sci., Vol. 7, No. 7, pp. 291-292, 2017.

[8] M. Kenteris, D. Gavalas, and D. Economou, "Mytilene E-guide: a multiplatform mobile application tourist guide exemplar", Multimed. Tools Appl., Vol. 54, No. 2, pp. 241-262, 2011.

[9] S. Nuraeni, A. Pradiva, and S. Novani, "Understanding Consumer Decision-Making in Tourism Sector : Conjoint Analysis", Procedia Soc. Behav. Sci., Vol. 169, No. August 2014, pp. 312-317, 2015.

[10] T. Anteneh Alemu, A. K. Tegegne, and A. Nega Tarekegn, "Recommender System in Tourism Using Case based Reasoning Approach", Int. J. Inf. Eng. Electron. Bus., Vol. 9, No. 5, pp. 3443, 2017.

[11] M. Farokhi, M. Vahid, M. Nilashi, and L. Branch, "A Multi-Criteria Recommender System for Tourism Using Fuzzy Approach", $J$. Soft Comput. Decis. Support Syst., Vol. 3, No. 4, pp. 19-29, 2016.

[12] I. M. A. Wirawan and I. W. B. Diarsa, "Mobilebased recommendation system for the tour package using the hybrid method", Int. J. Interact. Mob. Technol., Vol. 12, No. 8, pp. 6484, 2018.

[13] M. Figueredo, J. Ribeiro, N. Cacho, A. Thome, A. Cacho, F. Lopes, and V. Araujo, "From photos to travel itinerary: A tourism recommender system for smart tourism destination", In: Proc. of - IEEE 4th International Conf. on Big Data Computing Service and Applications, BigDataService 2018, pp. 85-92, 2018.

[14] Z. Bahramian, R. A. Abbaspour, and C. Claramunt, "A Cold Start Context-Aware Recommender System for Tour Planning Using Artificial Neural Network and Case Based Reasoning", Mob. Inf. Syst., Vol. 2017, No. 18, pp. 1-18, 2017.

[15] G. Hirakawa, G. Sato, K. Hisazumi, and Y. Shibata, "Data gathering system for recommender system in tourism", In: Proc. of 2015 18th International Conf. on NetworkBased Information Systems, pp. 521-525, 2015.

[16] D. Yaga, P. Mell, N. Roby, and K. Scarfone, Blockchain Technology Overview, National Institute of Standards and Technology, Gaithesburg, Maryland, USA, 2018.
[17] G. Zhang, T. Li, Y. Li, P. Hui, and D. Jin, "Blockchain-Based Data Sharing System for AIPowered Network Operations", J. Commun. Inf. Netw., Vol. 3, No. 3, pp. 1-8, 2018.

[18] M. A. Ferrag and L. Maglaras, "Deliverycoin: An IDS and blockchain-based delivery framework for drone-delivered services", Computers, Vol. 8, No. 3, pp. 1-15, 2019.

[19] C. Gao, X Chen, F. Feng, K. Zhao, X. He, Y.Li, and D. Jin, "Cross-domain recommendation without sharing user-relevant data", In: Proc. of The Web Conf. 2019 - In: Proc. of the World Wide Web Conf. WWW 2019, San Fransisco, USA, pp. 491-502, 2019.

[20] H. Drachsler, T. Bogers, R. Vuorikari, K. Verbert, E. Duval, N. Manouselis, G. Beham, S. Lindstaedth, H. Stern, M. Friedrich, and M. Wolpers, "Issues and considerations regarding sharable data sets for recommender systems in technology enhanced learning", Procedia Comput. Sci., Vol. 1, No. 2, pp. 2849-2858, 2010.

[21] R. M. Frey, D. Wörner, and A. Ilic, "Collaborative filtering on the blockchain: A secure recommender system for E-commerce", In: Proc. of AMCIS 2016: Surfing the IT Innovation Wave - 22nd Americas Conf. on Information Systems, San Diego, USA, pp. 1-5, 2016.

[22] T. F. Xue, Q.-C. Fu, C. Wang, and X.-Y. Wang, "A medical data sharing model via blockchain", Acta Autom. Sin., Vol. 49, No. 3, pp. 1555-1562, 2017.

[23] Q. Xia, E. B. Sifah, J. G. Kwame Omono Asamoah, X. Du, and M. Guizani, "MeDShare : Trust-less Medical Data Sharing Among", in IEEE Access, 2017, Vol. 5, pp. 1-10.

[24] M. Usman, "ScienceDirect Secure Electronic Medical Records Storage and Sharing Using Blockchain Technology", in Procedia Computer Science, 2020, Vol. 174, pp. 321-327.

[25] X. Liu, Z. Wang, C. Jin, F. Li, and G. Li, “A Blockchain-Based Medical Data Sharing and Protection Scheme", IEEE Access, Vol. 7, pp. 118943-118953, 2019.

[26] G. Zyskind, O. Nathan, and A. S. Pentland, "Decentralizing privacy: Using blockchain to protect personal data", Proc. - 2015 IEEE Secur. Priv. Work. SPW 2015, pp. 180-184, 2015.

[27] Y. Zhu, G. Zheng, and K. K. Wong, "Blockchain-empowered decentralized storage in air-to-ground industrial networks", IEEE Trans. Ind. Informatics, Vol. 15, No. 6, pp. 3593-3601, 2019. 
[28] T. Jiang, H. Fang, and H. Wang, "Blockchainbased internet of vehicles: Distributed network architecture and performance analysis", IEEE Internet Things J., Vol. 6, No. 3, pp. 4640-4649, 2019.

[29] C. Yu, X. Jiang, S. Yu, and C. Yang, "Blockchain-based shared manufacturing in support of cyber physical systems: concept, framework, and operation", Robot. Comput. Integr. Manuf., Vol. 64, No. December 2019, p. 101931, 2020.

[30] F. Peyghambari, M. Salehnia, M. F. Moghadam, M. R. Valujerdi, and E. Hajizadeh, "The correlation between the endometrial integrins and osteopontin expression with pinopodes development in ovariectomized mice in response to exogenous steroids hormones", Iran. Biomed. J., Vol. 14, No. 3, pp. 109-119, 2010.

[31] D. Buhalis, "Buhalis Marketing the competitive destination of the future", Tour. Manag., Vol. 21, No. January 1999, pp. 97-116, 2000.

[32] J. L. N. $\tilde{A}$ and F. J. Ma, "The influence of distance and prices on the choice of tourist destinations: The moderating role of motivations 's", Tour. Manag., Vol. 27, No. 5, pp. 982-996, 2006.

[33] D. Buhalis and A. Amaranggana, "Smart Tourism Destinations", Inf. Commun. Technol. Tour. 2014, pp. 553-564, 2014.

[34] Y. M. Arif, S. M. S. Nugroho, and M. Hariadi, "Selection of Tourism Destinations Priority using 6AsTD Framework and TOPSIS", in 2019 2nd International Seminar on Research of Information Technology and Intelligent Systems, ISRITI 2019, Yogyakarta, Indonesia pp. 346351, 2019.

[35] P. L. Lau and T. T. R. Koo, "Online popularity of destinations in Australia: An application of Polya Urn process to search engine data", $J$. Hosp. Tour. Manag., Vol. 42, No. February, pp. 277-285, 2020.

[36] S. Akbar and R. Hallak, "Identifying business practices promoting sustainability in aboriginal tourism enterprises in remote Australia", Sustain., Vol. 11, No. 17, pp. 1-18, 2019.

[37] S. Forouzandeh, A. R. Aghdam, M. Barkhordari, S. A. Fahimi, M. K. Fayqan, F. Forouzandeh, and E. G. Khani, "Recommender system for Users of Internet of Things (IOT)", IJCSNS Int. J. Comput. Sci. Netw. Secur., Vol. 17, No. 8, pp. 46-51, 2017.

[38] M. V. Gopalachari, "DBT recommender: Improved trustworthiness of ratings through debiasing tendency of users", Int. J. Intell. Eng. Syst., Vol. 11, No. 2, pp. 85-92, 2018.
[39] S. K. Gorakala, Building Recommendation Engine, Vol. 1, No. 1. Packt Publishing, 2016.

[40] G. Ha and Valerie Trifts, "Consumer Decision Making in Online Shopping Environments : The Effects of Interactive Decision Aids", Vol. 19, No. 1, pp. 4-21, 2000.

[41] M. Hassan, "Performance Analysis of Neural Networks-based Multi-criteria Recommender Systems", In: Proc. of 2017 2nd International Conf. on Information Technology, Information Systems and Electrical Engineering (ICITISEE), pp. 490-494, 2017.

[42] M. Hamada, N. B. Odu, and M. Hassan, "A fuzzy-based approach for modelling preferences of users in multi-criteria recommender systems", Proc. - 2018 IEEE 12th Int. Symp. Embed. Multicore/Many-Core Syst. MCSoC 2018, pp. 87-94, 2018.

[43] G. Adomavicius and Y. Kwon, Multi-Criteria Recommender Systems in Recommender Systems Handbooks, pp. 847-880, Springer Science+Business Media, New York, USA, 2015.

[44] M. Agathokleous and N. Tsapatsoulis, "Learning User Models in Multi-criteria Recommender Systems", Commun. Comput. Inf. Sci., Vol. 459 CCIS, pp. 205-216, 2014.

[45] A. Mikeli, D. Apostolou, and D. Despotis, "A multi-criteria recommendation method for interval scaled ratings", In: Proc. of - 2013 IEEE/WIC/ACM Int. Jt. Conf. Web Intell. Intell. Agent Technol. - Work. WI-IATW 2013, Vol. 3, pp. 9-12, 2013.

[46] A. Mikeli, D. Sotiros, D. Apostolou, and D. Despotis, "A multi-criteria recommender system incorporating intensity of preferences", In: Proc. of IISA 2013 - 4th Int. Conf. Information, Intell. Syst. Appl., pp. 231-234, 2013.

[47] J. Barreiro-Gomez and H. Tembine, "Blockchain token economics: A mean-fieldtype game perspective", IEEE Access, Vol. 7, pp. 64603-64613, 2019.

[48] H. F. Atlam, A. Alenezi, M. O. Alassafi, and G. B. Wills, "Blockchain with Internet of Things: Benefits, challenges, and future directions", Int. J. Intell. Syst. Appl., Vol. 10, No. 6, pp. 40-48, 2018.

[49] S. Figueroa, J. Añorga, and S. Arrizabalaga, "An attribute-based access control model in RFID systems based on blockchain decentralized applications for healthcare environments", Computers, Vol. 8, No. 3, pp. 1-19, 2019.

[50] Y. M. Arif, H. Nurhayati, S. Harini, S. M. Susiki Nugroho, and M. Hariadi, "Decentralized Tourism Destinations Rating System Using 
6AsTD Framework and Blockchain", In: Proc. of 2020 International Conf. on Smart Technology and Applications (ICoSTA), Surabaya, Indonesia, pp. 1-6, 2020.

[51] M. El Beqqal and M. Azizi, "Taxonomy on IoT technologies for designing smart systems", Int. J. Interact. Mob. Technol., Vol. 12, No. 5, pp. 182-191, 2018.

[52] W. Wang, D. T. Hoang, P. Hu, Z. Xiong, D. Niyato, P. Wang, Y. Wen, and D. I. Kim, "A Survey on Consensus Mechanisms and Mining Strategy Management in Blockchain Networks", IEEE Access, Vol. 7, No. c, pp. 22328-22370, 2019.

[53] E. Abstract, I. Bentov, C. Lee, and A. Mizrahi, "Proof of Activity: Extending Bitcoin's Proof of Work via Proof of Stake", ACM SIGMETRICS Perform. Eval. Rev., Vol. 42, No. 3, pp. 34-37, 2014. 